\title{
Band-Contact Lines in Electron Energy Spectrum of Graphite
}

\author{
G. P. Mikitik and Yu. V. Sharlai \\ B. Verkin Institute for Low Temperature Physics 83 Engineering, \\ Ukrainian Academy of Sciences, Kharkov 61103, Ukraine
}

(Dated: September 1, 2018)

\begin{abstract}
We discuss the known experimental data on the phase of the de Haas -van Alphen oscillations in graphite. These data can be understood if one takes into account that four band-contact lines exist near the $\mathrm{HKH}$ edge of the Brillouin zone of graphite.

PACS numbers: 71.18.+y, 03.65.Vf
\end{abstract}

\section{INTRODUCTION}

At present, graphite and its electronic properties attract considerable attention due to the discovery of novel carbon-based materials such as fullerenes and nanotubes constructed from wrapped graphite sheets,$\frac{1}{2}$ Besides, thin films of graphite give promise of device applications. ${ }^{2}$ The attention to graphite is also caused by specific features of its electron energy spectrum which result in interesting physical effects ${ }^{3.4}$ The electronic spectrum of graphite is described by the Slonzewski-Weiss-McClure (SWM) model,, .6 and values of the main parameters of this model were found sufficiently accurately from an analysis of various experimental data; see, e.g., the review of Brandt et $a l^{\underline{7}}$ and references therein. The Fermi surface of graphite consists of elongated pockets enclosing the edge HKH of its Brillouin zone ( see figures below). These pockets are formed by the two majority groups of electrons (e) and holes (h) which are located near the points $\mathrm{K}$ and $\mathrm{H}$ of the Brillouin zone, respectively. There is also at least one minority (m) low-concentration group of charge carriers in graphite, and this group seems to be located near the point $\mathrm{H}$. However, it is necessary to emphasize that in spite of the considerable attention attracted to graphite an unresolved problem concerned with its spectrum still exists.

It is well known ${ }^{5.6 .7}$ that in the edge HKH of the Brillouin zone of graphite, two electron energy bands are degenerate, and in a small vicinity of the edge these bands split linearly in a deviation of the wave vector $\mathbf{k}$ from the edge. In other words, the edge is the band-contact line. But, it was shown in our paper ${ }^{8}$ that if in the $\mathbf{k}$-space a closed semiclassical orbit of a charge carrier surrounds a contact line of its band with some other band (and lifting of the degeneracy is linear in $\mathbf{k}$ ), the wave function of this carrier after its turn over the orbit acquires the addition phase $\Phi_{B}= \pm \pi$ as compared to the case without the band-contact line. This $\Phi_{B}$ is the so-called Berry phase,,$\frac{9}{}$ and it modifies the constant $\gamma$ in the well-known semiclassical quantization rule $\frac{10}{\underline{\underline{T}}}$ for the energy $\varepsilon$ of a charge carrier in the magnetic fields $H$ :

$$
S\left(\varepsilon, k_{H}\right)=\frac{2 \pi e H}{\hbar c}(n+\gamma),
$$

where $S$ is the cross-sectional area of the closed orbit in the $\mathbf{k}$ space; $k_{H}$ is the component of $\mathbf{k}$ perpendicular to the plane of this orbit; $n$ is a large integer $(n>0) ; e$ is the absolute value of the electron charge, and the constant $\gamma$ is now given by the formula: $:^{\underline{8}}$

$$
\gamma=\frac{1}{2}-\frac{\Phi_{B}}{2 \pi} .
$$

When the magnetic field is applied along the HKH axis, orbits of electrons and holes in the Brillouin zone of graphite surround this axis. Thus, one might expect to find $\gamma=0$ for these orbits instead of the usual value $\gamma=1 / 2$ (the values $\gamma=0$ and $\gamma=1$ are equivalent).

A value of $\gamma$ can be measured using various oscillation effects and in particular, with the de Haas - van Alphen effect 11.12.13 For example, the first harmonic of the de Haas - van Alphen oscillations of the magnetic susceptibility has the form, 14

$$
\chi \cos \left(2 \pi \frac{\nu}{H}+\phi\right),
$$

where $\nu=\hbar c S_{e x} /(2 \pi e), S_{e x}$ is some extremal cross section of the Fermi surface of a metal in $k_{H}$, a positive $\chi$ is the amplitude of this first harmonic, and $\phi$ is its phase which is given by

$$
\phi=-2 \pi \gamma+\delta
$$

with $\delta= \pm \pi / 4$ for a minimum and maximum crosssection $S_{e x}$, respectively, and $\delta=0$ in the case of a two-dimensional Fermi surface $\frac{15}{15}$ It follows from Eq. (4) that one has to obtain $\phi_{e}=\phi_{h}=-\pi / 4$ for the maximum cross-sections of the electron and hole majorities in graphite. However, the phases $\phi_{e}, \phi_{h}$ measured long ago $\frac{16.17}{17}$ agree with the usual value $\gamma=0.5$; see Table I.

Recently a new method of determining the phase $\phi$ of the de Haas - van Alphen oscillations was elaborated, 15 and the authors of that paper found $\gamma=0$ for the cross section of the hole majority in graphite. However, in this determination they assumed the Fermi surface of the holes to be two-dimensional $\left(\delta_{h}=0\right)$; see Table I. Besides this, they found $\gamma=0.5$ for the maximum cross section of the electron majority, assuming the three-dimensional Fermi surface for this majority $\left(\delta_{e}=-\pi / 4\right)$. Although the obtained value $\gamma=0$ for the holes agrees with the above prediction, the results of Ref. 15 give rise to the following new problems: First, since the band-contact line in graphite penetrates both the electron and hole 


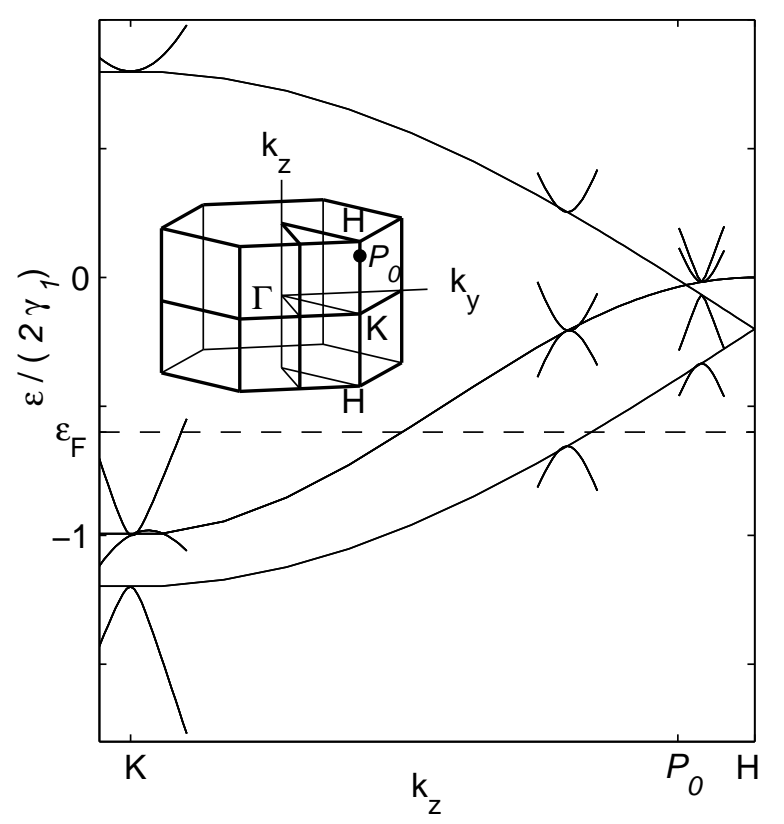

FIG. 1: Dependences of the bands $\varepsilon_{i}(i=1-4)$ on $\mathbf{k}$ near the edge $\mathrm{HKH}$ in graphite. Shown are the dependences $\varepsilon_{i}\left(k_{z}\right)$ at $k_{\perp}=0$ and the dependences of $\varepsilon_{i}$ on $k_{\perp}=\sqrt{k_{x}^{2}+k_{y}^{2}}$ at some characteristic values of $k_{z}$. The dashed line marks the position of the Fermi level $\varepsilon_{F}$. For clarity, in the construction of the figure the parameter $\gamma_{1}$ has been used which is twenty times smaller than that of Table II. For realistic value of $\gamma_{1}$ the point $P_{0}$ is much closer to the point $\mathrm{H}$ than in the figure. The insert shows the Brillouin zone of graphite and its characteristic points.

extremal cross sections, these cross sections must have the same $\gamma$. Second, using the values of the parameters of SWM model,,$\frac{7}{\underline{T}}$ one might expect that in graphite the electrons and holes of the extremal cross sections are both three-dimensional.

In this paper we show that in graphite, apart from the band-contact line coinciding with the edge $\mathrm{HKH}$, three additional band-contact lines exist near this edge. The existence of these lines leads to the usual value $\gamma=0.5$ for the maximum cross sections of the electron and hole majority groups in graphite. In other words, we resolve the above-mention contradiction between the theoretical value of $\gamma$ and the data of Refs. 16 17. We also discuss the data of Ref. 15 .

\section{BAND-CONTACT LINES IN GRAPHITE}

The SWM mode ${ }^{5.6}$ describes the wave-vector dependence of four electron energy bands of graphite $\varepsilon_{i}(\mathbf{k})$ $(i=1-4)$ in the vicinity of the vertical edge HKH of its Brillouin zone, Fig. 1. These bands can be found from the forth-order secular equation:

$$
\operatorname{det}|\hat{H}-\varepsilon|=0
$$

where the Hamiltonian matrix $\hat{H}$ has the form

$$
\hat{H}=\left(\begin{array}{cccc}
E_{1} & 0 & H_{13} & H_{13}^{*} \\
0 & E_{2} & H_{23} & -H_{23}^{*} \\
H_{13}^{*} & H_{23}^{*} & E_{3} & H_{33} \\
H_{13} & -H_{23} & H_{33}^{*} & E_{3}
\end{array}\right) .
$$

Here the following notations have been used:

$$
\begin{array}{r}
E_{1}=\Delta+\gamma_{1} \Gamma+\frac{1}{2} \gamma_{5} \Gamma^{2}, \\
E_{2}=\Delta-\gamma_{1} \Gamma+\frac{1}{2} \gamma_{5} \Gamma^{2}, \\
E_{3}=\frac{1}{2} \gamma_{2} \Gamma^{2}, \\
H_{13}=\frac{1}{\sqrt{2}}\left(-\gamma_{0}+\gamma_{4} \Gamma\right) \mathrm{e}^{i \alpha} \zeta, \\
H_{23}=\frac{1}{\sqrt{2}}\left(\gamma_{0}+\gamma_{4} \Gamma,\right) \mathrm{e}^{i \alpha} \zeta, \\
H_{33}=\gamma_{3} \Gamma \mathrm{e}^{i \alpha} \zeta,
\end{array}
$$

where $\alpha$ is the angle between the direction of the vector $\mathbf{k}$ and the $\Gamma K$ direction in the Brillouin zone; $\Gamma=$ $2 \cos \xi ; \xi$ and $\zeta$ are dimensionless wave vectors in the direction of the $z$-axis and in the basal plane, respectively: $\xi=(\pi / 2)\left(k_{z} /|K H|\right), \quad \zeta=(2 \pi / \sqrt{3})\left(k_{\perp} /|\Gamma K|\right)$; $k_{\perp}=\sqrt{k_{x}^{2}+k_{y}^{2}} ; \mathbf{k}$ is measured from the point $\mathrm{K}$. The parameter $\gamma_{0}$ which describes the interaction between neighbor atoms in a graphite layer is sufficiently large as compared to the other parameters $\gamma_{i}, \Delta$ which describe interactions between atoms in different graphite layers; see Table II. It is known ${ }^{7}$ that the band structure near the point $H$ is highly sensitive to values of the small parameters $\gamma_{2}$ and $\Delta$. While the value of $\gamma_{2}$ is known sufficiently well, the value of $\Delta$ was found less reliably ${ }^{7}$ Although our main conclusions remain unchanged for any reasonable $\Delta$, for definiteness, in subsequent analysis we shall use the set of the parameters ${ }^{7}$ based on the data of Refs. 161819,202122 and presented in Table II.

It is seen from Fig. 1 that two of the four bands are degenerate along the HKH edge of the Brillouin zone. In the interval from $\mathrm{K}$ to the point $P_{0}$ defined by the condition $E_{1}(\xi)=E_{3}(\xi)$ (i.e., by the equality $\cos \xi \approx$ $\left.|\Delta| / 2 \gamma_{1} \approx 0.01\right)$ these two bands are $\varepsilon_{2}(\mathbf{k})$ and $\varepsilon_{3}(\mathbf{k})$, while from $P_{0}$ to the point $\mathrm{H}$ they are $\varepsilon_{3}(\mathbf{k})$ and $\varepsilon_{4}(\mathbf{k})$. The contact of these bands is caused by the symmetry of the crystal. The change of the band degeneracy at $P_{0}$ can be understood if one takes into account a small spin-orbit interaction in SWM model (and then makes the interaction tend to zero). However, we emphasize here that as it follows from Eqs. (5) - (7), there are three additional contact lines of the same bands which are also located near the edge HKH, Fig. 2. The contact of the bands in these lines is accidental, ${ }^{23}$ i.e., is not caused by the symmetry of the crystal. It is due to the socalled trigonal warping ${ }^{7}$ of the Fermi surface which is characterized by the parameter $\gamma_{3}$. In the vicinity of the 
TABLE I: Frequencies $\nu$, phases $\phi$, and the appropriate $\gamma$ and $\delta$ of quantum oscillations in graphite for $S_{e}, S_{h}, S_{m}$ of Fig. 2

\begin{tabular}{|c|c|c|c|c|c|c|c|c|c|c|}
\hline & \multicolumn{4}{|c|}{$\mathrm{WFD}^{17}, 7$} & \multicolumn{4}{|c|}{$\mathrm{LK}^{15}$} & \multicolumn{2}{|c|}{ LK corrected } \\
\hline & $\nu(\mathrm{kOe})$ & $(\phi / \pi)$ & $(\delta / \pi)$ & $\gamma$ & $\nu(\mathrm{kOe})$ & $(\phi / \pi)$ & $(\delta / \pi)$ & $\gamma$ & $(\delta / \pi)$ & $\gamma$ \\
\hline $\bar{e}$ & $65 \pm 4$ & $0.64 \pm 0.18$ & $-1 / 4$ & $1 / 2$ & 46.8 & $3 / 4$ & $-1 / 4$ & $1 / 2$ & $-1 / 4$ & $1 / 2$ \\
\hline$h$ & $46 \pm 3$ & $0.76 \pm 0.1$ & $-1 / 4$ & $1 / 2$ & 64.1 & 1 & 0 & 0 & $-1 / 4$ & $3 / 8$ \\
\hline$m$ & $6 \pm 3$ & 0.06 & 0 & 0 & 3.28 & 0 & 0 & $1 / 2$ & 0 & 0 \\
\hline
\end{tabular}

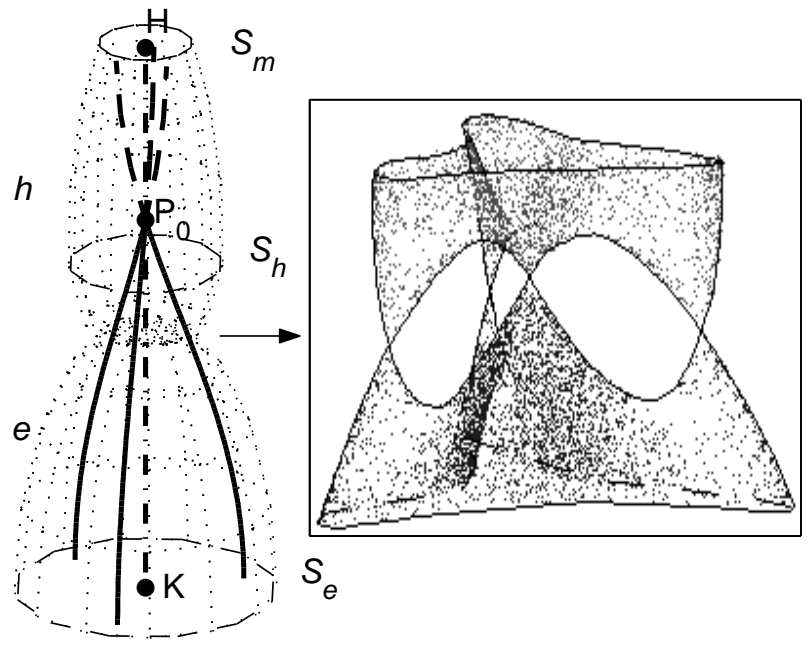

FIG. 2: Sketch of the Fermi surface (a half of it) and of the band contact lines in graphite. The accidental contact of the bands $\varepsilon_{2}(\mathbf{k})$ and $\varepsilon_{3}(\mathbf{k})$ occurs along the solid lines, while the dashed lines mark the accidental contact of the bands $\varepsilon_{3}(\mathbf{k})$ and $\varepsilon_{4}(\mathbf{k})$. The same bands are in contact along the HKH axis due to the symmetry of the crystal. All the lines merge at the point $P_{0}$. Shown are also the maximum cross sections of the electron $\left(S_{e}\right)$ and hole $\left(S_{h}\right)$ majorities and of the hole minority $\left(S_{m}\right)$ for the magnetic field along the HKH axis. A part of the Fermi surface where the electron and hole majorities touch is presented in an enlarged scale on the right; the band-contact lines pass through the conical features of the Fermi surface.

point $\mathrm{K}$ these lines can be approximately found from the equations:

$$
\sin 3 \alpha=-1, \quad \zeta \approx \frac{\sqrt{3}}{4} \frac{\gamma_{1} \gamma_{3}}{\gamma_{0}^{2}} \cos ^{2} \xi .
$$

Thus, the situation in graphite can be described as follows: The four contact lines of the bands $\varepsilon_{2}(\mathbf{k})$ and $\varepsilon_{3}(\mathbf{k})$ come to the point $P_{0}$ from the one side of the HKH axis, and the four contact lines of the bands $\varepsilon_{3}(\mathbf{k})$ and $\varepsilon_{4}(\mathbf{k})$ come to this point from the opposite side, and all these lines merge at the point $P_{0}$. It is essential that in the vicinity of all these lines the band splitting is linear in a deviation of $\mathbf{k}$ from the lines.

In graphite the electrons of the band $\varepsilon_{3}(\mathbf{k})$ give rise to the majority electron group, while the holes of the band $\varepsilon_{2}(\mathbf{k})$ make up the hole majority. All the contact lines
TABLE II: Values of the parameters $(\mathrm{eV})$ of the SWM model ${ }^{7}$

\begin{tabular}{rr|cr}
\hline \hline$\gamma_{0}$ & $3.16 \pm 0.05$ & $\gamma_{4}$ & $0.044 \pm 0.024$ \\
$\gamma_{1}$ & $0.39 \pm 0.01$ & $\gamma_{5}$ & $0.038 \pm 0.005$ \\
$\gamma_{2}$ & $-0.020 \pm 0.002$ & $\Delta$ & $-0.008 \pm 0.002$ \\
$\gamma_{3}$ & $0.315 \pm 0.015$ & $\varepsilon_{F}$ & $-0.024 \pm 0.002$ \\
\hline \hline
\end{tabular}

of the bands $\varepsilon_{2}(\mathbf{k})$ and $\varepsilon_{3}(\mathbf{k})$ lie under the Fermi surface of these majority groups. When the lines pass from the electron part of the surface to its hole part, the conical features of the Fermi surface, the so-called "outrigger"

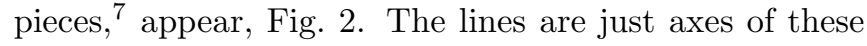
four pieces connecting the electron and hole parts.

As was shown in our paper $\stackrel{24}{=}$ in the magnetic field $H$ parallel to a band-contact line the splitting of the Landau levels for the electron states near the line is proportional to $\sqrt{H}$ rather than to $H$. In the case of graphite these levels are known as the so-called "leg levels" investigated by Dresselhaus ${ }^{25}$ The existence of the leg levels (and, in fact, of the band-contact lines in graphite) was confirmed by magneto-optical experiments ${ }^{20.26}$ Interestingly, the well-known large diamagnetism of graphite ${ }^{27}$ is also caused by electron states near the band-contact lines ${ }^{28}$

\section{DISCUSSION}

When the magnetic field $H$ is directed along the $z$ axis, the maximum electron cross section in $k_{z}$ is located at $\xi=0$, while the maximum cross section of the hole majority is between the points $\mathrm{K}$ and $P_{0}$, viz., at $\cos \xi \approx \pm\left(\varepsilon_{F} / 6 \gamma_{2}\right)^{1 / 2} \approx 0.45$ where $\varepsilon_{F}$ is the Fermi energy in graphite, see Fig. 2. Thus, both these cross sections are penetrated by the four band-contact lines. However, an even number of the band-contact lines do not change $e^{8,29}$ the usual value $\gamma=1 / 2$. Thus, we find $\gamma=1 / 2$ for the maximum cross sections of the majority groups, which agrees with the experimental results of Refs. 1617.

We now discuss briefly the value of $\gamma$ for the minority group. For the parameters presented in Table II, the hole minority is located near the point $\mathrm{H}$ and it results from the band $\varepsilon_{1}(\mathbf{k})$. At this point the minority and the hole majority produced by the band $\varepsilon_{2}(\mathbf{k})$ have equal cross sections when the magnetic field is along the $\mathrm{HKH}$ 
axis. Since no contact lines of the bands $\varepsilon_{1}(\mathbf{k})$ and $\varepsilon_{2}(\mathbf{k})$ penetrate this common cross section, one might expect to find the usual value $\gamma=1 / 2$ in this case. However, the semiclassical approximation which is used in deriving Eqs. (1) and (2) fails for the hole orbits corresponding to this cross section since for this approximation to be valid, the orbits must be sufficiently far away from each other. The analysis carried out beyond the scope of the semiclassical approximation ${ }^{17}$ led to $\gamma=0$ and $\delta=0$ for the "degenerate" orbit. In experiments this orbit is ascribed to the hole minority, and the phase $\phi_{m}$ measured in Ref. 17 agrees with these $\gamma$ and $\delta$, see Table I.

In Ref. 15 a new method was developed to determine the phase $\phi$ of the de Haas -van Alphen oscillations of the magnetic susceptibility. The appropriate results for $\phi$ and $\gamma$ in graphite are presented in Table I. However, authors of Ref. 15 implied in their analysis of $\gamma$ that the sign of $\chi$ in formula (3) is positive in the case of electrons and negative for holes. This is not correct; the sign is always positive. A re-examination of the derivation of the Lifshits-Kosevich formula 14 proves this statement 30 With this in mind we have corrected $\delta$ and $\gamma$ of Ref. 15 , and the obtained results are also presented in Table I.

For the hole minority and for the electron majority 31 the corrected results coincide with those of Williamson et $a l l^{17}$ (but $\delta_{m}=0$ can be caused by the above-mentioned degeneracy of the hole orbits rather than by the twodimensional spectrum of the hole minority). For the hole majority the phases $\phi_{h}$ measured in Refs. 17 and 15 disagree. The phase $\phi_{h}=\pi$ obtained by Luk'yanchuk and Kopelevich $^{15}$ means that either the spectrum of these carriers is two dimensional, or if $\delta=-\pi / 4$, one obtains $\gamma=3 / 8$. However, in the semiclassical approxi- mation, $\gamma$ can be equal to $1 / 2$ or to 0 only ${ }^{8,11}$ Intermediate values can occur in situations close to the magnetic breakdown ${ }^{32}$ In principle, such the situation is possible for the SWM model, but it does not occur for the parameters presented in Table II.

The parameters of Table II correspond to three dimensional spectrum of graphite and lead to a consistent description of the experimental data16.18.19.20.21.22 obtained many yeas ago. However, Luk'yanchuk and Kopelevich ${ }^{15}$ used the highly oriented pyrolytic graphite (HOPG) with very high ratio of the out-of-plane to basal-plane resistivities $\left(\sim 5 \times 10^{4}\right)$, and in this sample, quantum-Hall-effect features were observed which indicate a quasi two dimensional nature of this $\mathrm{HOPG}^{3}$ It was also $\operatorname{argued}^{33}$ that in similar samples of HOPG an incoherent transport occurs in the direction perpendicular to the graphite layers, and the three dimensional spectrum of carriers seems to fail. If this conclusion is valid only for the hole majority, it could explain the above-mentioned disagreement. This also means that the parameters of SWM model should be reconsidered to describe the spectrum of such HOPG.

To conclude, the phases of the de Haas - van Alphen oscillations in graphite were measured in Refs. 1516 17. The data of Refs. 1617 can be completely explained in the framework of the known band structure of graphite ${ }^{7}$ if one takes into account that four band-contact lines exist near the HKH edge of its Brillouin zone. The data of Luk'yanchuk and Kopelevich ${ }^{15}$ obtained for HOPG disagree with the experimental results of Refs. 1617 for one of the two large cross sections and probably imply that a reconsideration of the energy-band parameters for such $\mathrm{HOPG}$ is required.
1 R. Saito, G. Dresselhaus, and M. S. Dresselhaus, Physical properties of Carbon Nanotubes (Imperial College Press, London, 1998).

2 K. S. Novoselov, A. K. Geim, S. V. Morozov, S. V. Dubonos, Y. Zhang, D. Jiang, cond-mat/0410631 (unpublished).

3 Y. Kopelevich, J. H. S. Torres, R. R da Silva, F. Mrowka, H. Kempa, P. Esquinazi, Phys. Rev. Lett. 90, 156402 (2003).

${ }^{4}$ K. S. Novoselov, A. K. Geim, S. V. Morozov D. Jiang, M. I. Katsnelson, I. V. Grigorieva, S. V. Dubonos, A. A. Firsov, Nature 438, 197 (2005).

5 J. C. Slonzewski, P. R. Weiss, Phys. Rev. 109(2), 272 (1958).

6 J. W. McClure, Phys. Rev. 108, 612 (1957).

7 N. B. Brandt, S. M. Chudinov, and Ya. G. Ponomarev, Semimetals I. Graphite and its Compouds (Elsevier, Amsterdam, 1988).

8 G. P. Mikitik, Yu. V. Sharlai, Phys. Rev. Lett. 82, 2147 (1999).

9 M. V. Berry, Proc. Roy. Soc. London A 392, 45 (1984).

10 D. Shoenberg, Magnetic Oscillations in Metals (Cambridge University Press, Cambridge, England, 1984).
11 G. P. Mikitik and Yu. V. Sharlai, Zh. Eksp. Teor. Fiz. 114, 1375 (1998) [Sov. Phys. JETP 87, 747 (1998)].

12 G. P. Mikitik, Yu. V. Sharlai, Phys. Rev. B 65, 184426 (2002).

13 G. P. Mikitik, Yu. V. Sharlai, Phys. Rev. Lett. 93, 106403 (2004).

14 I. M. Lifshits and A. M. Kosevich, Zh. Eksp. Teor. Fiz. 29, 730 (1955) [Sov. Phys. JETP 2, 636 (1956)].

15 I. A. Luk'yanchuk, Y. Kopelevich, Phys. Rev. Lett. 93, 166402 (2004).

16 D. E. Soule, J. W. McClure, L. B. Smith, Phys. Rev. 134, A453 (1964).

17 S. J. Williamson, S. Foner, M. S. Dresselhaus, Phys. Rev. 140, A1429 (1965).

18 W.V. Toy, M.S. Dresselhaus, G. Dresselhaus, Phys. Rev. B 15, 4077 (1977).

19 A. Misu, E. Mendez, M.S. Dresselhaus, J. Phys. Soc. Jpn. 47, 199 (1979).

20 R.E. Doezema, W.R. Datars, H. Schaber, A. Van Schyndel, Phys. Rev. B 19, 4224 (1979).

21 E. Mendez, T.C. Chien, N. Kambe, M.S. Dresselhaus, Solid State Commun. 33, 837 (1980).

22 E. Mendez, A. Misu, M.S. Dresselhaus, Phys. Rev. B 
21(2), 827 (1980)

23 C. Herring, Phys. Rev. 52, 365 (1937).

24 G. P. Mikitik and Yu. V. Sharlai, Fiz. Nizk. Temp. 22, 762 (1996) [Low Temp. Phys. 22, 585 (1996)].

25 G. Dresselhaus, Phys. Rev. B 10, 3602 (1974).

${ }^{26}$ D.A. Platts, D.D.L. Chung, M.S. Dresselhaus, Phys. Rev. B 15, 1087 (1977).

27 J.W. McClure, Phys. Rev. 101, 1642 (1956); ibid 119, 606 (1960); M.P. Sharma, L.C. Johnson, J.W. McClure, Phys. Rev. B 9, 2467 (1974).

28 G. P. Mikitik, I. V. Svechkarev, Fiz. Nizk. Temp. 15, 295 (1989) [Sov. J. Low Temp. Phys. 15, 165 (1989)].

29 Although each line adds 0.5 to $\gamma$, but within the semiclas- sical approximation $\gamma$ is defined up to an integer.

30 Note that for holes, the integration $\int_{0}^{\infty} d n$ in Ref. 14 is rearranged to the integration over the energy from 0 to $-\infty$ rather than from 0 to $\infty$.

31 Note that in Ref. 15 the frequencies $\nu$ for the electron and hole majorities are interchanged as compared to popular belief ${ }^{7}$; see Table I.

32 M. Ya. Azbel', Zh. Eksp. Teor. Fiz. 39, 1276 (1960) [Sov. Phys. JETP 12, 891 (1961)]; A. A. Slutskin, Zh. Eksp. Teor. Fiz. 53, 767 (1967).

33 H. Kempa, H. C. Semmelhack, P. Esquinazi, Y. Kopelevich, Solid State Commun. 125, 1 (2003). 